\title{
Experimental Observation of the Blob-Generation Mechanism from Interchange Waves in a Plasma
}

\author{
I. Furno, ${ }^{1}$ B. Labit, ${ }^{1}$ M. Podestà, ${ }^{1}$ A. Fasoli, ${ }^{1}$ S. H. Müller,${ }^{1,2}$ F. M. Poli, ${ }^{1}$ P. Ricci,,${ }^{1}$ C. Theiler, ${ }^{1}$ \\ S. Brunner, ${ }^{1}$ A. Diallo, ${ }^{1}$ and J. Graves ${ }^{1}$ \\ ${ }^{1}$ Centre de Recherches en Physique des Plasmas-Ecole Polytechnique Fédérale de Lausanne, \\ Association EURATOM-Confédération Suisse, CH-1015 Lausanne, Switzerland \\ ${ }^{2}$ Center for Energy Research, University of California, San Diego, La Jolla, California 92093, USA
}

(Received 16 June 2007; published 6 February 2008)

\begin{abstract}
The mechanism for blob generation in a toroidal magnetized plasma is investigated using time-resolved measurements of two-dimensional structures of electron density, temperature, and plasma potential. The blobs are observed to form from a radially elongated structure that is sheared off by the $\mathbf{E} \times \mathbf{B}$ flow. The structure is generated by an interchange wave that increases in amplitude and extends radially in response to a decrease of the radial pressure scale length. The dependence of the blob amplitude upon the pressure radial scale length is discussed.
\end{abstract}

In a variety of magnetic confinement devices, such as tokamaks [1-3], stellarators [4], reversed field pinches [5], and linear devices [6-8], intermittent cross-field transport of particles and energy are associated, at least in part, with the propagation of plasma blobs. These are poloidally localized regions extending along the field lines where the plasma pressure is enhanced compared to the surrounding plasma. How blobs are generated remains an open question.

In this Letter, we investigate experimentally the dynamics of blobs and identify a generation mechanism on the TORPEX toroidal device [9]. In TORPEX, blobs are observed to originate at the crest of a coherent interchange wave [10]. A simple magnetic configuration is used with both toroidal and vertical field components. Low temperature and density plasmas allow measurements of local parameters with high spatial and temporal resolution across the entire plasma cross section. The generation mechanism is studied using time-resolved two-dimensional (2D) profiles of electron density and temperature, plasma potential, and $\mathbf{E} \times \mathbf{B}$ velocity, which are conditionally sampled over many blob events. Blobs are observed to form from radially elongated structures that are sheared off by the $\mathbf{E} \times \mathbf{B}$ flow. These structures develop from an interchange wave that increases in amplitude and extends radially in response to a decrease of the radial pressure scale length. We discuss the dependence of the blob amplitude upon the pressure radial scale length. These results detail a fundamental phenomenon in plasmas and can be used to validate theories and numerical simulations of blob dynamics [11]. Similarly to the tokamak scrape-off layer (SOL), the magnetic configuration features open field lines, a $\nabla B$, and magnetic field curvature. Blobs in TORPEX exhibit universal statistical properties with strong similarities with observations in the tokamak SOL [12]. Thus, the observed dynamics may shed light on the blob ejection mechanism in tokamaks, where there are strong indications that blobs result from interchange instabilities in the SOL [13].

In the TORPEX (major radius $R=1 \mathrm{~m}$, minor radius $a=0.2 \mathrm{~m}$ ) experiments investigated here, hydrogen plasmas are produced and sustained by microwaves in the electron cyclotron range of frequencies [14]. A microwave power of $\approx 400 \mathrm{~W}$ is used in a toroidal magnetic field of $76 \mathrm{mT}$ on axis and a vertical magnetic field $B_{z}=2.3 \mathrm{mT}$, which results in a vertically elongated plasma configuration. The plasma source is localized on the high field side (HFS), with negligible plasma production for $r \geq 5 \mathrm{~cm}$ [14]. Figure 1 shows $2 \mathrm{D}$ profiles of the time-averaged (indicated by the overbar symbol) electron pressure $\bar{p}_{e}=$ $\bar{n}_{e} \bar{T}_{e}$, plasma potential $\bar{V}_{\mathrm{pl}}$, and the $\mathbf{E} \times \mathbf{B}$ velocity, $\overline{\mathbf{v}}_{E \times B}$, obtained from Langmuir probe measurements. The plasma potential is computed from $\bar{V}_{\mathrm{pl}}=\bar{V}_{\mathrm{fl}}+\mu \bar{T}_{e} / e$, where $\bar{V}_{\mathrm{fl}}$ and $\bar{T}_{e}$ are floating potential and electron temperature, and the coefficient $\mu=3.1 \pm 0.6$ is determined experimen-

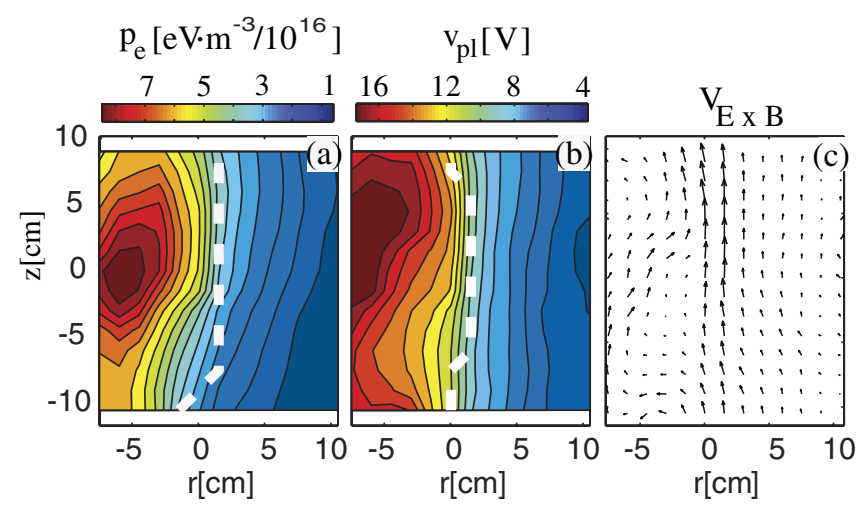

FIG. 1 (color). 2D poloidal profiles of the time-averaged plasma pressure (a), plasma potential (b), and $\mathbf{E} \times \mathbf{B}$ velocity (c) from Langmuir probe measurements. Dashed lines indicate the position of (a) maximum $\left|\bar{L}_{\mathrm{pe}}^{-1}\right|$ and (b) maximum of the radial gradient of the plasma potential. 
tally [14]. The $\mathbf{E} \times \mathbf{B}$ velocity is evaluated from $\overline{\mathbf{v}}_{E \times B}=$ $\left(-\nabla \bar{V}_{\mathrm{pl}} \times \mathbf{B}\right) / B^{2}$.

The profiles of pressure and plasma potential peak in the HFS region and decrease toward the low field side (LFS). The $\overline{\mathbf{v}}_{E \times B}$ field in Fig. 1(c) is almost vertical for $r \gtrsim 0$ with a $z$ component, $\bar{v}_{z, E \times B}$, which is radially sheared. The inverse radial pressure gradient scale length $\left|\bar{L}_{\mathrm{pe}}^{-1}\right|=$ $\left|\nabla_{r} \bar{p}_{e}\right| / \bar{p}_{e}$ has a maximum on a vertical line at $r \approx$ $1 \mathrm{~cm}$; see Fig. 1(a). Within the experimental uncertainty, this corresponds to the position of the maximum $\left|\bar{v}_{z, E \times B}\right|$, represented by a dashed line in Fig. 1(b). On this line at the midplane, $\bar{n}_{e} \approx 8 \times 10^{15} \mathrm{~m}^{-3}, \quad \bar{T}_{e} \approx 3.7 \mathrm{eV},\left|\bar{L}_{\mathrm{pe}}^{-1}\right| \approx$ $15 \mathrm{~m}^{-1}$, and $\bar{v}_{z, E \times B} \approx 1200 \mathrm{~ms}^{-1}$. The $\overline{\mathbf{v}}_{E \times B}$ shear is maximum on a vertical line at $r \approx 3 \mathrm{~cm}$, where $\left|\partial \bar{v}_{z, E \times B} / \partial r\right| \approx 3 \times 10^{4} \mathrm{~s}^{-1}$ at the midplane.

In this configuration, similarly to the scenario investigated in Ref. [10], we identify two distinct poloidal regions with different plasma dynamics: (1) a main plasma region for $r \leq 5 \mathrm{~cm}$, where the plasma source is localized, dominated by a coherent interchange wave for $-5 \lesssim r \lesssim 5 \mathrm{~cm}$; (2) a LFS region for $r \geqslant 5 \mathrm{~cm}$ with negligible plasma production and broadband fluctuation spectra, dubbed source-free region, characterized by the propagation of blobs.

In Figs. 2(a) and 2(b), we illustrate the nature of the fluctuations in the two regions using 2D profiles of skewness $S$ and kurtosis $K$ (normalized third and fourth order moments of the probability density function) of ionsaturation signals. In the main plasma region for $-5 \leqslant$ $r \lesssim 5 \mathrm{~cm}$, fluctuations are characterized by coherent oscillations at a frequency of $\sim 3.9 \mathrm{kHz}$ as shown by an ionsaturation signal in Fig. 2(c). In Fig. 2(a), the 2D profile of the spectral power in the range $f=3.9 \pm 1 \mathrm{kHz}$ peaks around the position of maximum $\left|\bar{L}_{\mathrm{pe}}^{-1}\right|$, where such range represents approximately $50 \%$ of the total spectral power of the fluctuating signal. At this position, $S \sim 0$ and $K \sim 2$, consistently with the presence of coherent oscillations. A vertical wave number $k_{z} \approx 30.6 \pm 3 \mathrm{~m}^{-1}$ and an upper bound for the parallel wave number $k_{\|}^{\max } \approx 0.046 \mathrm{~m}^{-1} \ll$ $k_{z}$ are measured using a two-point correlation technique [15]. The wave propagates upward with a velocity of $\sim 1200 \mathrm{~ms}^{-1}$, consistent with the time-averaged $\overline{\mathbf{v}}_{E \times B}$ profile in Fig. 1(c). The wave is identified as an interchange mode by solving a linearized Hasegawa-Wakatani model [16], taking the magnetic field curvature and gradient into account together with the measured time-averaged density and $\overline{\mathbf{v}}_{E \times B}$ profiles. This mode is found to be unstable, with fastest growth rate for $k_{\|}=0$. For $k_{z}=30.6 \mathrm{~m}^{-1}$, the real frequency of the mode is $\sim 4 \mathrm{kHz}$, in agreement with the experimental value.

In the source-free region, the fluctuation spectrum is broad and exempt from coherent modes. The skewness is positive $(S \sim 1)$ indicating the occurrence of intermittent bursts as shown in Fig. 2(d). Figure 2(e) shows the mean velocity field of positive structures identified by the condition $I_{\text {sat }}-\bar{I}_{\text {sat }}>4.5 \mathrm{~mA}$ and lasting between 40 and
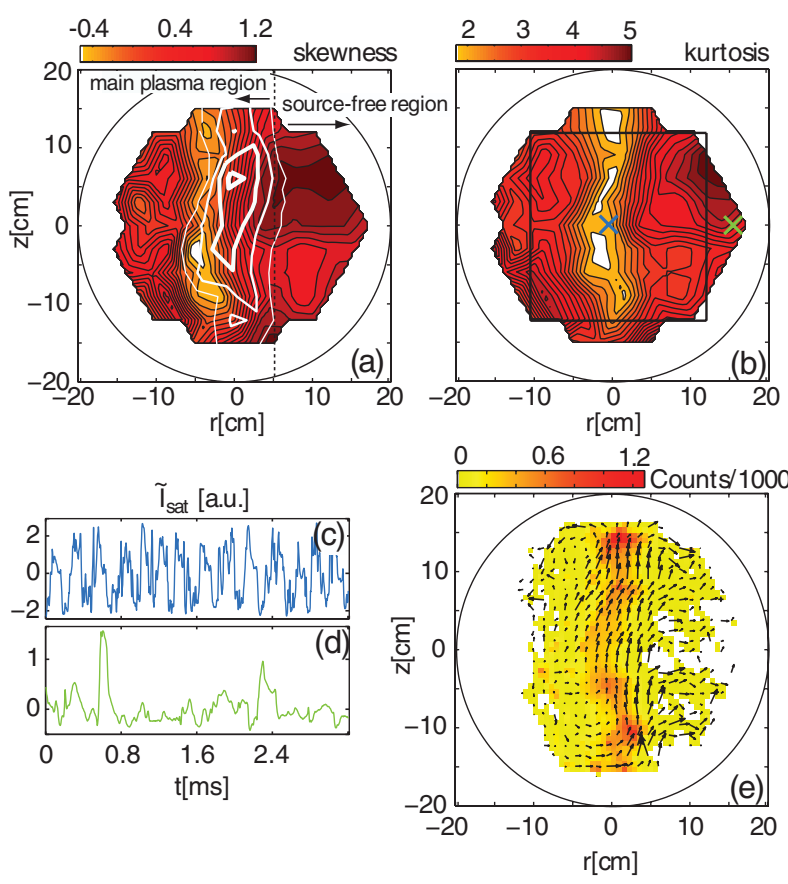

FIG. 2 (color). Poloidal profiles of (a) skewness and (b) kurtosis of ion-saturation signals. In (a) the profile of spectral power in the frequency range $3.9 \pm 1 \mathrm{kHz}$ is shown in white and localizes the interchange wave. Contours correspond to $90 \%$, $60 \%, 30 \%$, and $10 \%$ of the maximum spectral power. The generation mechanism is investigated in the rectangle in (b). (c),(d) Ion-saturation signals at the two locations indicated in (b) show coherent fluctuations in the main plasma region (blue cross) and intermittent bursts at the edge (green cross). (e) Mean velocity field of positive structures and histogram (in color) of trajectory counts.

$200 \mu \mathrm{s}$, as evaluated by a spatiotemporal pattern recognition method [17]. The velocity pattern is not sensitive to the choice of the parameters used to identify the structures. The pattern suggests that the bursts are associated with the presence of blobs that originate in the main plasma region and propagate outward into the source-free region as individual coherent structures over distances of the order of the minor radius.

To investigate the mechanism for blob generation, timeresolved 2D profiles of $n_{e}, T_{e}, V_{\mathrm{pl}}$ and velocity fields are required. These are obtained by performing a conditional sampling [18] over many blob events of the $I-V$ characteristic of a Langmuir probe in a time window centered around the blob detection. The Langmuir probe voltage is swept at $1 \mathrm{kHz}$ frequency and signals are acquired at $250 \mathrm{kHz}$. A reference probe, located at $r=14 \mathrm{~cm}, z=0$ and toroidally displaced by $90^{\circ}$ from the Langmuir probe, measures a reference ion-saturation signal $I_{\text {ref }}$. We perform a selection of blobs over eight different classes of amplitudes defined by the conditions $(1+j / 2) \sigma \leq \tilde{I}_{\text {ref }} \leq$ $(1.2+j / 2) \sigma$ with $j=0,1,2, \ldots, 7$, where $\tilde{I}_{\text {ref }}=$ $I_{\text {ref }}-\bar{I}_{\text {ref }}$ is the fluctuating signal (in the following all fluctuating quantities are denoted by the overtilde symbol) 
and $\sigma$ is its standard deviation over the whole discharge with $\sigma / \bar{I}_{\text {ref }} \approx 0.45$. As detailed below, this is used to investigate the link between the blob amplitude and the pressure scale length. Since blobs are not synchronous with the sweeping signal, the full conditionally sampled $I-V$ probe characteristic can be reconstructed $[14,19]$ which is then used to extract $n_{e}, T_{e}$, and $V_{\mathrm{pl}}$. The upper bound of the overall uncertainties for these quantities is estimated as $30 \%$ using error propagation analysis. By moving the probe between discharges over a $25 \times 20 \mathrm{~cm}^{2}$ section in the $r$ - $z$ plane, Fig. 2(b), we obtain 2D profiles with $1 \mathrm{~cm}$ radial and $1.8 \mathrm{~cm}$ vertical resolution, from which the $\mathbf{E} \times$ B velocity profile is reconstructed.

For the class of blobs defined by $4.5 \sigma \leq \tilde{I}_{\text {ref }} \leq 4.7 \sigma$, Fig. 3 illustrates the results of the conditional sampling of $\approx 600$ blobs over three identical $2.7 \mathrm{~s}$ long plasma discharges. Figure 3(a) shows the time evolution of $\tilde{n}_{e}$ in the mode region (red) and in the source-free region (black). Figures 3(b)-3(e) show 2D profiles of $\tilde{n}_{e}$ at four different times during the ejection of the blob together with the total $\mathbf{E} \times \mathbf{B}$ velocity field. The coherent structures in Figs. 3(b) and 3(c) are identified with the interchange wave, based on their spatiotemporal properties.

The dynamics of blob formation and ejection from the interchange wave is captured by frames (c)-(e). A radially
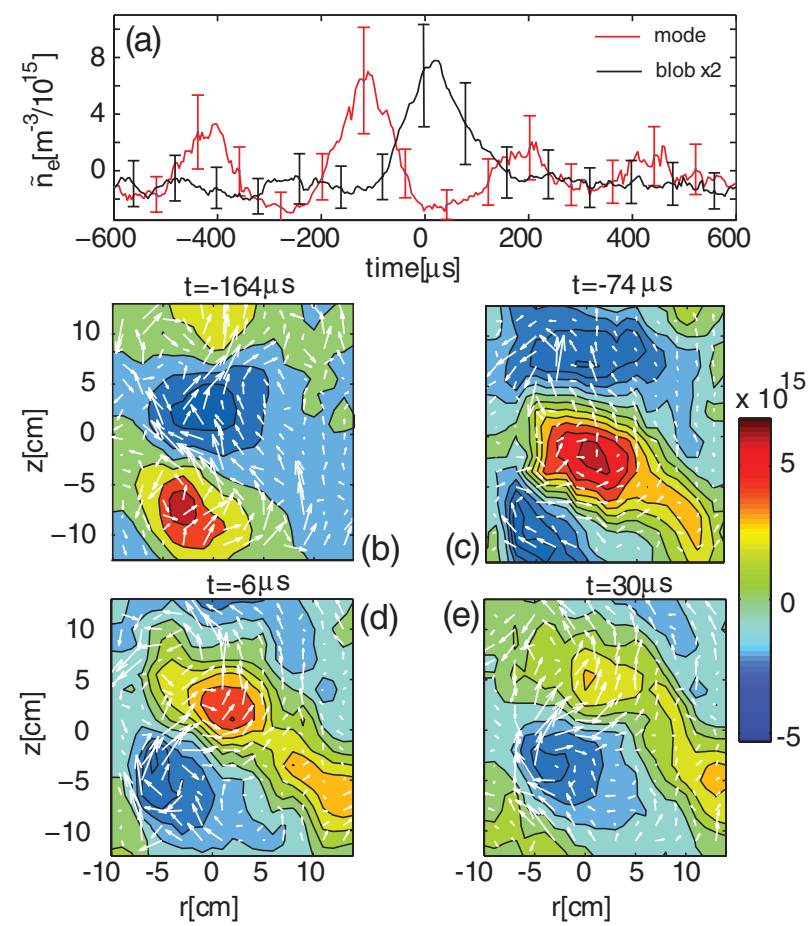

FIG. 3 (color). Plasma dynamics from the conditional sampling technique. (A full animation is provided in Ref. [20].) Shown are (a) time history of $\tilde{n}_{e}$, including uncertainties, in the mode region (red) at $r=-1 \mathrm{~cm}, z=-5 \mathrm{~cm}$ and in the sourcefree region (black) at $r=13 \mathrm{~cm}, z=-5 \mathrm{~cm}$. Note that the latter is multiplied by two for clarity. (b)-(e) 2D profiles of $\tilde{n}_{e}$ at different times during blob ejection. The arrows show the instantaneous $\mathbf{v}_{E \times B}$ profile. elongated density structure forms from the positive cell of the wave, Fig. 3(c). The formation of this structure follows from the convection of plasma by the $\mathbf{E} \times \mathbf{B}$ flow in a corridor that extends radially over several ion sound radii $\left(\rho_{s} \approx 0.3 \mathrm{~cm}\right)$, Fig. 3(c). In this corridor, the velocity is mainly in the radial direction and is dominated by the $\mathbf{E} \times$ B velocity. In Fig. 3(e) for $t=-6 \mu \mathrm{s}$, the elongated density structure is convected upward in a sheared velocity field that moves HFS and LFS parts of the density structure with different vertical velocities. A relative displacement between them is obtained, Fig. 3(d). Eventually, the original density structure breaks into two parts, Fig. 3(e), and the new structure on the low field side forms a plasma blob.

The role of the $\mathbf{E} \times \mathbf{B}$ flow in shearing off the density structure can be quantified by computing the shearing time [21] $1 / \tau_{\mathrm{sh}}=\left(k_{z} L_{r} / 2 \pi\right) \partial v_{z, E \times B} / \partial r$, where $L_{r}$ is the radial width of the shear layer and $k_{z}$ is the wave number in the $z$ direction. Both $L_{r}$ and $v_{z, E \times B}$ are estimated by averaging the instantaneous values over the trajectory of the positive cells. For the data set considered here, using $L_{r} \approx 0.03 \mathrm{~m}$, $k_{z} \approx 30.6 \mathrm{~m}^{-1}$, and $\left(\partial v_{z, E \times B}\right) / \partial r \approx 3 \times 10^{4} \mathrm{~s}^{-1}$, we find $\tau_{\mathrm{sh}} \approx 200 \mu \mathrm{s}$. The shearing time $\tau_{\mathrm{sh}}$ is of the same order of the transit time $\tau_{\text {tr }}=2 \pi /\left(k_{z} v_{z, E \times B}\right) \approx 200 \mu \mathrm{s}$ during which a density structure moves upward by $2 \pi / k_{z}$. These estimates confirm that the $\mathbf{E} \times \mathbf{B}$ flow can shear the density structure that forms from the wave.

In Fig. 4, we illustrate the mechanism driving the elongation of the density wave crest. In Fig. 4(a), a zoomed view of the instantaneous pattern of the fluctuating $\tilde{\mathbf{v}}_{E \times B}$ is shown together with the total pressure contours. The interchange mechanism that exchanges a zone of high plasma pressure with a zone of low plasma pressure is clearly at play. The local radial pressure gradient driving the interchange instability is maximum in the region where the pressure negative wave crest is localized, Fig. 4(a). The time evolution of $\left|L_{\mathrm{pe}}^{-1}\right|$ in the wave frame at the position of maximum intensity is shown in Fig. 4(c) together with that of the mode amplitude, Fig. 4(b). The latter is evaluated at
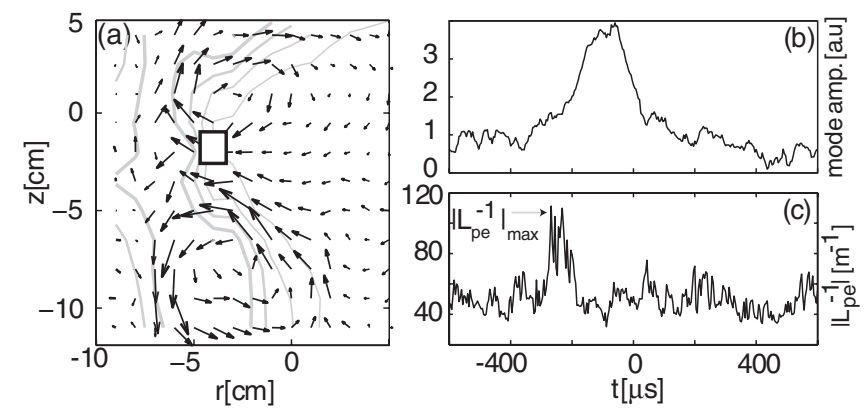

FIG. 4. (a) A zoomed view of the instantaneous fluctuating $\mathbf{E} \times \mathbf{B}$ velocity field at $t=-184 \mu$ s shows the convective cells interchanging zones of high and low plasma pressure. Solid gray lines represent equispaced pressure contours with $\Delta p_{e}=$ $10^{16} \mathrm{~m}^{-3} \mathrm{eV}$. Time evolution of (b) mode amplitude and (c) inverse pressure gradient length $\left|L_{\mathrm{pe}}^{-1}\right|$ at the point of maximum observed intensity, indicated by the square in (a). 


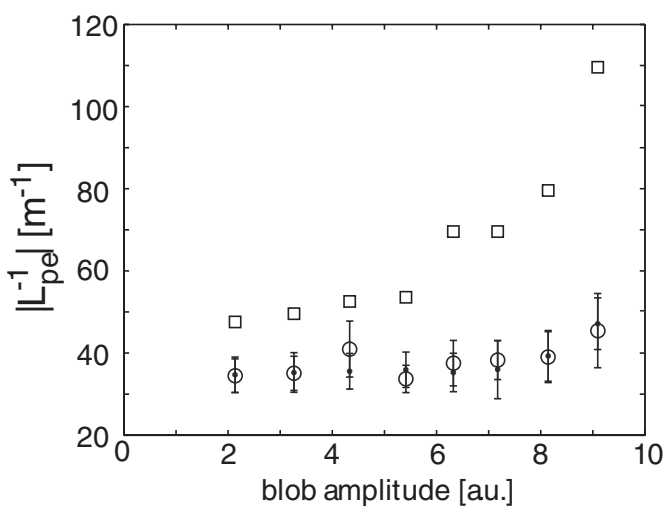

FIG. 5. Dependence of various inverse pressure gradient scale lengths upon the blob size for the eight blob classes. Squares represent $\left|L_{\mathrm{pe}}^{-1}\right|_{\max }$. Circles and dots are, respectively, the timeaveraged values of $\left|L_{\mathrm{pe}}^{-1}\right|$ before and after the blob event.

each time step as $\sqrt{\sum_{k_{r}}\left|\tilde{n}_{e}\left(k_{r}, k_{z 0}\right)\right|^{2}}$, where $\tilde{n}_{e}\left(k_{r}, k_{z 0}\right)$ is the 2D spatial Fourier transform evaluated for the dominant mode $k_{z 0} \approx 30 \mathrm{~m}^{-1}$ and $k_{r}$ is the radial wave number. During a first period, $-600 \mu \mathrm{s}<t<-350 \mu \mathrm{s}$, both mode amplitude and $\left|L_{\mathrm{pe}}^{-1}\right|$ remain approximately constant. Later in time, $\left|L_{\mathrm{pe}}^{-1}\right|$ is observed to increase at $t \approx$ $-350 \mu \mathrm{s}$ from $\left|L_{\mathrm{pe}}^{-1}\right| \approx 40 \mathrm{~m}^{-1}$ to a maximum value $\left|L_{\mathrm{pe}}^{-1}\right|_{\max } \approx 110 \mathrm{~m}^{-1}$ at $t \approx-260 \mu \mathrm{s}$. This is followed by an increase of the mode amplitude which leads to a larger radial $\mathbf{v}_{E \times B}$ and to the elongation of the positive wave crest.

In order to investigate the link between the blob amplitude and the pressure scale length, we perform the conditional sampling analysis over the eight different classes of blob amplitudes previously defined. For each class, the temporal evolution of $\left|L_{\mathrm{pe}}^{-1}\right|$ and mode amplitude is similar to Fig. 4. Figure 5 shows the dependence upon the blob amplitude of $\left|L_{\mathrm{pe}}^{-1}\right|_{\max }$ and of the averaged values of $\left|L_{\mathrm{pe}}^{-1}\right|$ in a $200 \mu \mathrm{s}$ time window centered, respectively, $400 \mu \mathrm{s}$ before and after the blob event. These values correspond to a stationary gradient before and after the blob ejection and do not show a strong dependence upon the blob amplitude. This suggests that the blob removes the excess of gradient and brings the profile back to the starting condition. A monotonic and faster than linear dependence of $\left|L_{\mathrm{pe}}^{-1}\right|_{\max }$ upon the blob amplitude is found, showing that the ejection of blobs is preceded by an increase of the pressure gradient that leads to blobs with larger amplitude for larger $\left|L_{\text {pe }}^{-1}\right|_{\max }$.

In summary, we have identified experimentally a mechanism for blob generation from interchange waves in a simple, magnetized toroidal device. The results show that when a blob is generated the following sequence of events occurs: (1) the local pressure gradient length decreases, providing an increase of the drive for the interchange mode; (2) the mode increases in amplitude and radially expands; (3) the radially elongated structure is sheared off by the $\mathbf{E} \times \mathbf{B}$ flow, forming the blob.

The present results represent an important test bed for validating both theoretical models and simulations of blob dynamics. In tokamak geometry, a link between a local increase of the radial pressure gradient and the blob formation has recently been predicted for the edge region [22]. The identification of the blob generation mechanism may open new venues for controlling edge turbulent transport.

This work is partly supported by the Fonds National Suisse de la Recherche Scientifique. P. R. is supported by a Euratom Fusion grant. We thank F. Skiff for useful discussions.

[1] J. Boedo et al., Phys. Plasmas 8, 4826 (2001); J. Boedo et al., Phys. Plasmas 10, 1670 (2003).

[2] S. Zweben, Phys. Fluids 28, 974 (1985).

[3] S. Krasheninnikov, Phys. Lett. A 283, 368 (2001).

[4] R. Sanchez et al., Phys. Rev. Lett. 90, 185005 (2003).

[5] M. Spolaore et al., Phys. Rev. Lett. 93, 215003 (2004).

[6] G. Antar et al., Phys. Rev. Lett. 87, 065001 (2001).

[7] T. Carter, Phys. Plasmas 13, 010701 (2006).

[8] T. Windisch, O. Grulke, and T. Klinger, Phys. Plasmas 13, 122303 (2006).

[9] A. Fasoli et al., Bull. Am. Phys. Soc. 13, 119 (2003); A. Fasoli et al., Phys. Plasmas 13, 055902 (2006).

[10] S. H. Müller et al., Phys. Plasmas 14, 110704 (2007).

[11] N. Bisai et al., Phys. Plasmas 11, 4018 (2004); O.E. Garcia et al., Plasma Phys. Controlled Fusion 48, L1 (2006).

[12] B. Labit et al., Phys. Rev. Lett. 98, 255002 (2007); B. Labit et al., Plasma Phys. Controlled Fusion 49, B281 (2007).

[13] J. Myra et al., Phys. Plasmas 13, 092509 (2006).

[14] M. Podestà et al., Plasma Phys. Controlled Fusion 47, 1989 (2005).

[15] F. M. Poli et al., Phys. Plasmas 13, 102104 (2006).

[16] A. Hasegawa and M. Wakatani, Phys. Rev. Lett. 50, 682 (1983).

[17] S. H. Müller et al., Phys. Plasmas 13, 100701 (2006).

[18] H. Johnsen et al., Phys. Fluids 30, 2239 (1987); H. Pecseli and J. Trulsen, Phys. Fluids B 1, 1616 (1989).

[19] T. Pierre et al., Phys. Rev. Lett. 92, 065004 (2004).

[20] See EPAPS Document No. E-PRLTAO-100-052801 for a complete movie of the blob dynamics. For more information on EPAPS, see http://www.aip.org/pubservs/ epaps.html.

[21] H. Biglari, P. Diamond, and P. Terry, Phys. Fluids B 2, 1 (1990).

[22] S. I. Krasheninnikov and A. I. Smolyakov, Phys. Plasmas 14, 102503 (2007). 